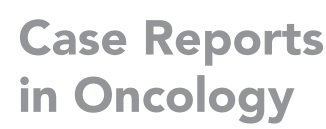

\title{
A Rare Chromosome Abnormality with der(16)t(1;16)(q12;q11.2) in Blast Crisis of Chronic Myeloid Leukemia
}

\author{
Ryo Yanagiyaa, b Daisuke Ishikawa ${ }^{c}$ Tomomi Toubai ${ }^{b}$ Tsubasa Ichikawa ${ }^{a}$ b \\ Naofumi Kawaguchic ${ }^{c}$ Kunie Sugasawa ${ }^{b}$ Kenichi Ishizawa ${ }^{a}$ Soichi Saito ${ }^{b}$ \\ aDepartment of Neurology, Hematology, Metabolism, Endocrinology and Diabetology \\ (3rd Internal Medicine), Faculty of Medicine, Yamagata University, Yamagata, Japan; \\ bepartment of Internal Medicine, Nihonkai General Hospital, Sakata, Japan; \\ 'Department of Pharmacy, Nihonkai General Hospital, Sakata, Japan
}

\author{
Keywords \\ Chronic myeloid leukemia - Blast crisis - Additional chromosomal abnormality · \\ $\mathrm{t}(1 ; 16)(\mathrm{q} 12 ; \mathrm{q} 11.2) \cdot$ Genetic instability
}

\begin{abstract}
Although tyrosine kinase inhibitors markedly improve the clinical outcome of chronic myeloid leukemia (CML), blast crisis in $\mathrm{CML}(\mathrm{CML}-\mathrm{BC})$ still has a poor prognosis. Many chromosomal abnormalities have been reported in CML-BC and may contribute to therapeutic resistance, disease progression, and prognosis. Herein, we report a rare chromosome abnormality with $\operatorname{der}(16) t(1 ; 16)(q 12 ; q 11.2)$ in CML-BC. It has been demonstrated that this chromosomal abnormality is associated with disease progression and poor prognosis in other malignancies, such as Ewing sarcoma. A 70-year-old man with CML who had been treated with imatinib and dasatinib was admitted to our hospital after complaining for several weeks of fatigue and dyspnea and diagnosed with CML-BC. His tumor cells presented additional chromosomal abnormality with $\operatorname{der}(16) t(1 ; 16)(q 12 ; q 11.2)$, which has never been reported in $C M L$ cases. We successfully treated him using cytotoxic agents combined with ponatinib, and this chromosome abnormality was detected via G-banding. Our patient has lived for over 8 months without any progression with ponatinib treatment alone. Although the biological function of this chromosomal abnormality remains unclear, the satellite DNA of 1q12, which induces genomic instability in other malignancies, and the loss of $16 q$ may contribute to the disease progression of CML in this case. In conclusion, this paper is the first to report on the case of CML-BC with $\operatorname{der}(16) t(1 ; 16)(q 12 ; q 11.2)$.


Yanagiya et al.: CML-BC with $\operatorname{der}(16) t(1 ; 16)(q 12 ; q 11.2)$

\section{Introduction}

Chronic myeloid leukemia (CML) is characterized by the translocation of chromosome 9q34 Abl1 and chromosome 22q11.2 Bcr in hematopoietic stem cells [1]. Abl1 is a protooncogene that encodes a tyrosine kinase and is involved in the promotion of the cell cycle [2]. Abnormal tyrosine kinases encoded by $\mathrm{t}(9 ; 22)(\mathrm{q} 34 ; \mathrm{q} 11.2) B c r / A b l 1$ form a tetramer via the coiled-coil region encoded by $B c r$, thereby activating $A b l 1$-encoding tyrosine kinases permanently [3]. Activated Abl1-encoding tyrosine kinases induce disorder in the cell cycle and are responsible for the pathogenesis of chronic CML (CML-CP) [1]. Tyrosine kinase inhibitors (TKIs) inhibit the ATP-binding portion of Abl1-encoding tyrosine kinases and are capable of effectively reducing $B c r / A b l 1$-positive cells in CML-CP patients. There are currently several TKIs (imatinib, nilotinib, dasatinib, bosutinib, and ponatinib) available worldwide [4], and the development of these TKIs has resulted in a dramatic improvement in the prognosis of CML-CP. The 5-year overall survival (OS) of newly-diagnosed CML-CP patients who show a good response to TKIs is over $90 \%[5,6]$. However, patients who are refractory or intolerant to TKIs continue to show a poor prognosis [5, 6]. Blast crisis in CML (CML-BC) is characterized by excessive blast proliferation accompanied by differentiation impairment or extramedullary lesion [7] and has an extremely poor prognosis. The median OS of CML-BC patients treated with TKIs is approximately 12 months, while the median failure-free survival is around 5 months [8]. There is no standard therapeutic strategy for CML-BC. Although allogeneic hematopoietic stem cell transplantation is the only curative therapeutic option for CML-BC, the clinical outcome remains poor due to high rates of relapse and treatment-related mortality [8]. The pathogenesis of CML-BC remains unclear; however, additional chromosomal abnormalities may contribute to the progression of CML-BC [9].

Herein, we report on a CML-BC patient with chromosomal abnormalities in addition to der(16)t $(1 ; 16)(q 12 ; q 11.2)$. Contrary to previous reports that this chromosomal abnormality is associated with a poor prognosis [10], we succeeded in controlling the patient's disease status using TKIs. This patient has not experienced any disease progression for over 8 months.

\section{Case Report/Case Presentation}

A 70-year-old man was admitted to our hospital after complaining for several weeks of fatigue and dyspnea. He was diagnosed with CML-CP 14 years ago and was initially treated with imatinib $400 \mathrm{mg} /$ day. He achieved major molecular response (MMR) within a year of initiating treatment with imatinib, but decided to discontinue treatment due to economic reasons after 80 months while still with MMR. Although this patient lost MMR 4 months after discontinuing imatinib, he refused to restart treatment. Twenty-seven months after stopping imatinib, he also lost his major cytogenetic response and started treatment with dasatinib (180 mg/day). Although the patient regained MMR within 7 months of initiating treatment, he presented uncontrollable pleural effusion as a result of treatment with dasatinib and was switched onto imatinib (400 mg/day). His disease status remained well controlled for 54 months, but deteriorated gradually before being diagnosed with blast crisis in October 2019. The patient's laboratory findings at CML-BC diagnosis are shown in Table 1 . The blasts in his peripheral blood (PB) were 90\% and showed hypercoagulation and a high uremic acid concentration in the serum. In addition, bone marrow aspiration showed excessive blast proliferation (more than $80 \%$ of all nucleated cellular counts). Flow cytometry analysis showed that the blasts were positive for CD13, CD19, and CD10, but negative for CD33, CD14, CD11c, CD3, CD4, CD8, and cytoplasmic myeloperoxidase, which suggests B-cell phenotype (B-lymphoid BC). Real-time polymerase chain reaction (RT-PCR) for $b c r / a b l 1$ of PB showed

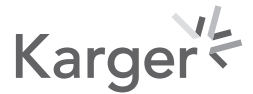




\section{Case Reports in Oncology}

Table 1. Laboratory findings at admission

\begin{tabular}{l|l}
\hline Case Rep Oncol 2020;13:1020-1025 \\
\hline DOI: 10.1159/000509642 & $\begin{array}{l}\text { ○ 2020 The Author(s). Published by S. Karger AG, Basel } \\
\text { www.karger.com/cro }\end{array}$ \\
\hline
\end{tabular}

\begin{tabular}{ll}
\hline Complete blood cell counts & \\
WBC & $287,060 / \mu \mathrm{L}$ \\
Blast & $90 \%$ \\
Segment & $2 \%$ \\
Band & $2 \%$ \\
Mono & $0.5 \%$ \\
Lymp & $5.5 \%$ \\
RBC & $204 \times 10^{4} / \mu \mathrm{L}$ \\
Hb & $6.3 \mathrm{~g} / \mathrm{dL}$ \\
Ht & $21.4 \%$ \\
Plt & $5.6 \times 10^{4} / \mu \mathrm{L}$ \\
Retic & $1.35 \%$ \\
\hline Coagulation & \\
PT & $75.1 \%$ \\
APTT & $26.1 \mathrm{~s}$ \\
Fibrinogen & $367 \mathrm{mg} / \mathrm{dL}$ \\
FDP & $21.5 \mu \mathrm{g} / \mathrm{dL}$ \\
D-dimer & $6.84 \mu \mathrm{g} / \mathrm{dL}$ \\
AT III & $89 \%$ \\
\hline Biochemistry & \\
TP & \\
Alb & $6.3 \mathrm{~g} / \mathrm{dL}$ \\
T.Bil & $4 \mathrm{~g} / \mathrm{dL}$ \\
AST & $0.7 \mathrm{mg} / \mathrm{dL}$ \\
ALT & $26 \mathrm{U} / \mathrm{L}$ \\
LD & $12 \mathrm{U} / \mathrm{L}$ \\
ALP & $929 \mathrm{U} / \mathrm{L}$ \\
$\gamma$ GT & $236 \mathrm{U} / \mathrm{L}$ \\
BUN & $78 \mathrm{U} / \mathrm{L}$ \\
Cr & $14.4 \mathrm{mg} / \mathrm{dL}$ \\
UA & $0.92 \mathrm{mg} / \mathrm{dL}$ \\
Na & $9.6 \mathrm{mg} / \mathrm{dL}$ \\
K & $142 \mathrm{mEq} / \mathrm{L}$ \\
Cl & $4.6 \mathrm{mEq} / \mathrm{L}$ \\
Ca & $109 \mathrm{mEq} / \mathrm{L}$ \\
P & $8 \mathrm{mg} / \mathrm{dL}$ \\
CRP & $2.1 \mathrm{mg} / \mathrm{dL}$ \\
& $8.81 \mathrm{mg} / \mathrm{dL}$ \\
\hline & \\
& \\
\hline & \\
\hline
\end{tabular}

WBC, white blood cells; Mono, monocyte; Lymp, lymphocyte; RBC, red blood cells; Hb, hemoglobin; Ht, hematocrit; Plt, platelet counts; Retic, reticulocyte; PT, prothrombin time; APTT, activated partial thromboplastin time; FDP, fibrin/fibrinogen degradation products; AT III, antithrombin III; TP, total protein; Alb, albumin; T.Bil, total bilirubin; AST, aspartate aminotransferase; ALT, alanine aminotransferase; LD, lactate dehydrogenase; ALP, alkaline phosphatase; $\gamma \mathrm{GT}$, gammaglutamyl transferase; BUN, blood urea nitrogen; $\mathrm{Cr}$, creatinine; $\mathrm{UA}$, uric acid; CRP, C-reactive protein.

$1.3 \times 10^{5}$ copies/ $\mu$ g RNA. As a result, the patient was diagnosed with B-lymphoid crisis. Interestingly, the patient was found to have an additional chromosome abnormality with 46,XY,t(9;22)(q34;q11.2)[3]/46,idem, der(4)t(1;4)(q12;q35)[1]/46,idem,der(16)t(1;16) (q12;q11.2),i(18)(q10)[1] in PB. The spectral karyotyping of the blasts in the PB demonstrated that all the cells (4 cells) showed reciprocal translocation of chromosomes 9 and 22. In addition, two of the 4 cells analyzed showed translocation of fragments derived from chro- 


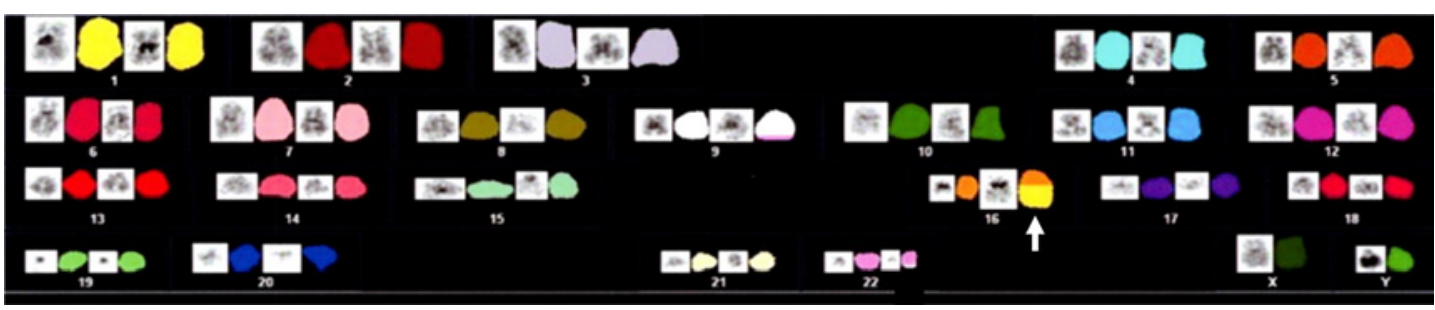

Fig. 1. Spectral karyotyping of peripheral blasts showing the translocation of the fragment from chromosome 1 to chromosome 16 (arrow) and reciprocal translocation of chromosomes 9 and 22.

mosomes 1 to 16 (Fig. 1). This additional chromosomal abnormality was not detected at the initial diagnosis.

The patient's symptoms quickly worsened, and he developed disseminated intravascular coagulopathy (DIC). He was started on treatment with $1 \mathrm{mg} / \mathrm{kg} /$ day of prednisolone accompanied with 24-hour continuous intravenous cytarabine $100 \mathrm{mg} /$ body/day, $380 \mathrm{U} / \mathrm{kg} /$ day of recombinant thrombomodulin alfa, and transfusion with fresh frozen plasma for DIC. After cytarabine treatment, the patient's blasts in PB gradually decreased and his coagulation status improved. We decided to increase the dosage of cytarabine to $100 \mathrm{mg} / \mathrm{m}^{2} /$ day, in addition to $50 \mathrm{mg} /$ body of daunorubicin. His blasts disappeared from the PB 15 days after initiating chemotherapy. The patient was then started on ponatinib (15 mg/day). Bone marrow aspiration was performed after chemotherapy and showed a decrease in blasts (3.6\% of nucleated cellular counts). Fluorescence in situ hybridization of $b c r$ and $a b l 1$ showed that the patient achieved major cytogenetic response (fusion signal was positive for $4 \%$ of 100 assessed cells). However, G-banding of the bone marrow blasts still showed 46,XY,del(6) (q?),t(9;22)(q34;q11.2)[1]/46,XY[19]. The patient's overall condition was markedly improved and he was subsequently discharged. He continued to receive treatment with $15 \mathrm{mg} /$ day of ponatinib and has not yet experienced disease progression, as of June 2020. His international scale of RT-PCR for $b c r / a b l 18$ months after starting ponatinib was $0.2319 \%$. The patient achieved an optimal response at the time, according to European LeukemiaNet 2013. His laboratory findings did not show a decrease in normal hematopoiesis or excessive blast proliferation from the time of his discharge.

\section{Discussion/Conclusion}

According to previous studies, increased genetic instability and incomplete repairment of deoxynucleic acids caused by Bcr/Abl1 plays an important role in generating chromosomal abnormalities in CML cells [10]. Mitelman reported that "major" and "minor" pathways of generating additional chromosomal abnormalities exist in CML cells [11]. For example, trisomy 8, i(17q), trisomy 19, and an extra Ph are generated via the "major" pathway, and about $70 \%$ of CML cases have at least one of these four chromosomal abnormalities [11]. On the other hand, $-7,-17,+17,+21,-Y$, and $t(3 ; 21)(q 26 ; q 22)$ were generated via the "minor" pathway, while about $15 \%$ of CML cases had at least one of these six chromosomal abnormalities [11]. Asnafi et al. reviewed the additional chromosomal abnormalities discovered in CML-BC cases between 1975 and 2017 [9] and suggested that some chromosomal abnormalities are associated with poor prognosis and poor therapeutic responses to TKIs [9]. However, the impact of these additional chromosomal abnormalities on the development of a blast crisis remains unknown and detailed investigation will be required in the future. 
In our patient, der(16)t(1;16)(q12;q11.2) was identified by both G-banding and spectral karyotyping assays. There are few reports available on CML patients with this abnormal chromosome on PubMed or Atlas of Genetics and Cytogenetics in Oncology and Hematology, a database of chromosomal abnormalities in malignant tumors (http://atlasgeneticsoncology. org/; accessed June 19, 2020). This chromosomal abnormality has been reported in several types of malignancies, including breast cancer, multiple myeloma, Wilms tumor, and Ewing sarcoma (http://atlasgeneticsoncology.org/; accessed June 19, 2020) and has been associated with disease progression and poor clinical outcome $[12,13]$. Although the biological function of $\operatorname{der}(16) \mathrm{t}(1 ; 16)(\mathrm{q} 12 ; \mathrm{q} 11.2)$ remains unclear, a previous report suggested that the translocation of 1q12 satellite DNA can mediate epigenetic perturbations and genetic instability [14]. In addition to 1q12 translocation, an unbalanced loss of chromosome 16 may also contribute to promote disease progression and a relatively poor prognosis in malignant tumors. Although the function of the unbalanced loss of $16 \mathrm{q}$ remains unclear, according to a previously reported retrospective analysis, both 1q gain and 16q loss, including unbalanced translocation $\mathrm{t}(1 ; 16)(\mathrm{q} 10 \sim 21 ; \mathrm{q} 10 \sim 13)$, may be related to 5-year OS in cases of Ewing sarcoma [15]. In regards to the poor prognosis of $\operatorname{der}(16) \mathrm{t}(1 ; 16)(\mathrm{q} 12 ; \mathrm{q} 11.2)$, our patient showed relatively better responses to chemotherapy and ponatinib compared with the reported failure-free survival of CML-BC treated with TKIs [8]. Intriguingly, we found that this additional chromosomal abnormality disappeared after chemotherapy. The long-term therapeutic response will have to be carefully monitored, since this chromosomal abnormality has been previously reported as a poor prognostic factor $[12,13,15]$. In summary, this paper is the first to report on the case of a CML-BC patient with $\operatorname{der}(16) \mathrm{t}(1 ; 16)(\mathrm{q} 12 ; \mathrm{q} 11.2)$. Further CML cases with additional chromosomal abnormalities related to $1 \mathrm{q}$ or $16 \mathrm{q}$, as well as abnormal chromosomes and other associated gene mutations, will need to be analyzed to improve our understanding of the pathophysiology of additional chromosomal abnormalities in CML.

\section{Acknowledgment}

We thank all staffs working in hematology and nephrology unit of Nihonkai General Hospital for their good medical, psychiatric, and social support for this patient.

\section{Statement of Ethics}

Informed consent was obtained from our patient. This study is in compliance with the ethical requirements of our institutions (Nihonkai General Hospital and Yamagata University).

\section{Conflict of Interest Statement}

The authors have no conflicts of interest to declare related to this case report.

\section{Funding Sources}

This study did not receive any funding.

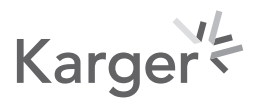




\section{Case Reports in Oncology}

\begin{tabular}{l|l}
\hline Case Rep Oncol 2020;13:1020-1025 \\
\hline DOI: 10.1159/000509642 & $\begin{array}{l}\text { @ 2020 The Author(s). Published by S. Karger AG, Basel } \\
\text { www.karger.com/cro }\end{array}$ \\
\hline
\end{tabular}

Yanagiya et al.: CML-BC with $\operatorname{der}(16) \mathrm{t}(1 ; 16)(\mathrm{q} 12 ; \mathrm{q} 11.2)$

\section{Author Contributions}

Ryo Yanagiya, Tsubasa Ichikawa, Kunie Sugasawa, and Soichi Saito treated the patients during his admission. Ryo Yanagiya and Soichi Saito selected the therapeutic strategies. Ryo Yanagiya wrote the article and has final responsibility. Soichi Saito supervised the hospital staff during the patient's admission. Daisuke Ishikawa and Naofumi Kawaguchi provided the patient and staff with pharmacological support. Tomomi Toubai and Kenichi Ishizawa supervised Ryo Yanagiya during the treatment of the patient and the writing of this article.

\section{References}

1 Apperley JF. Chronic myeloid leukaemia. Lancet. 2015;385(9976):1447-59.

2 Goldman JM, Melo JV. Targeting the BCR-ABL tyrosine kinase in chronic myeloid leukemia. N Engl J Med. 2001; 344(14):1084-6.

3 McWhirter JR, Galasso DL, Wang JY. A coiled-coil oligomerization domain of Bcr is essential for the transforming function of Bcr-Abl oncoproteins. Mol Cell Biol. 1993;13(12):7587-95.

4 Shanmuganathan N, Treatment of chronic myeloid leukemia: assessing risk, monitoring response, and optimizing outcome. Leuk Lymphoma. 2017;58(12):2799-810.

5 Ross JE, Saglio G, Kantarjian HM, Baccarani M, Mayer J, Boqué C, et al. Final 5-Year Study Results of DASISION: The Dasatinib Versus Imatinib Study in Treatment-Naïve Chronic Myeloid Leukemia Patients Trial. J Clin Oncol. 2016;34(20):2333-40.

6 Hochhaus A, Saglio G, Hughes TP, Larson RA, Kim DW, Issaragrisil S, et al. Long-term benefits and risks of frontline nilotinib vs imatinib for chronic myeloid leukemia in chronic phase: 5-year update of the randomized ENESTnd trial. Leukemia. 2016;30(5):1044-54.

7 Vardiman JW, Harris NL, Brunning RD. The World Health Organization (WHO) classification of the myeloid neoplasms. Blood 2002;114(5):937-51.

8 Jain P, Kantarjian HM, Ghorab A, Sasaki K, Jabbour EJ, Gonzalez GN, et al. Prognostic Factors and Survival Outcomes in Patients With Chronic Myeloid Leukemia in Blast Phase in the Tyrosine Kinase Inhibitor Era: Cohort Study of 477 Patients. Cancer. 2017;123(22):4391-402.

9 Asnafi AA, Deris Zayeri Z, Shahrabi S, Zibara K, Vosughi T. Chronic Myeloid Leukemia With Complex Karyotypes: Prognosis and Therapeutic Approaches. J Cell Physiol. 2019;234(5):5798-806.

10 Slupianek A, Poplawski T, Jozwiakowski SK, Cramer K, Pytel D, Stoczynska E. et al. BCR/ABL stimulates WRN to promote survival and genomic instability. Cancer Res. 2011;71(3):842-51.

11 Mitelman F. The Cytogenetic Scenario of Chronic Myeloid Leukemia. Leuk Lymphoma. 1993;11(Suppl 1): 11-5.

12 Hattinger CM, Rumpler S, Ambros IM, Strehl S, Lion T, Zoubek A, et al. Demonstration of the translocation $\operatorname{der}(16) t(1 ; 16)(q 12 ; q 11.2)$ in interphase nuclei of Ewing tumors. Genes Chromosomes Cancer. 1996;17(3): 141-50.

13 Hattinger CM, Zoubek A, Ambros PF. Molecular Cytogenetics in Ewing Tumors: Diagnostic and Prognostic Information. Onkologie. 2000;23(5):416-22.

14 Fournier A, McLeer-Florin A, Lefebvre C, Duley S, Barki L, Ribeyron J, et al. 1q12 chromosome translocations form aberrant heterochromatic foci associated with changes in nuclear architecture and gene expression in $B$ cell lymphoma. EMBO Mol Med. 2010;2(5):159-71.

15 Hattinger CM, Pötschger U, Tarkkanen M, Squire J, Zielenska M, Kiuru-Kuhlefelt S, et al. Prognostic Impact of Chromosomal Aberrations in Ewing Tumours. Br J Cancer. 2002;86(11):1763-9. 